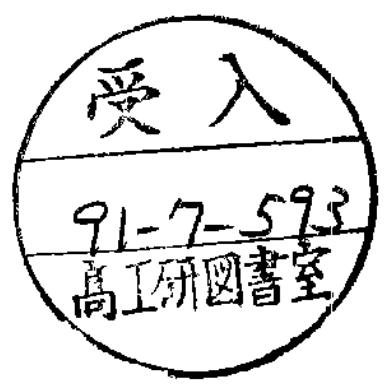

$\mathrm{ACT}-36$

CERN-TH-6100/91

CTP-TAMU-31/91

\title{
SUPERSYMMETRY BREAKING AND DARK MATTER
}

Jorge L. Lopez ${ }^{\star}$ and Kajia Yuan

Center for Theoretical Physics, Department of Physics

Texas A\&M University, College Station, TX 77843-4242, U.S.A.

and

Astroparticle Physics Group, Houston Advanced Research Center (HARC)

The Woodlands, TX 77381, U.S.A.

and

D.V. Nanopoulos ${ }^{\dagger}$

Center for Theoretical Physics, Department of Physics

Texas A \& M University, College Station, TX 77843-4242, U.S.A.

and

Astroparticle Physics Group

Houston Advanced Research Center (HARC)

The Woodlands, TX 77381, U.S.A.

and

Theoretical Physics Division

CERN, CH 1211 Geneva 23

\begin{abstract}
We explore the possibility of neutralino dark matter in minimal SUSY GUTs models. We emphasize the use of accurate formulae for the squark and slepton masses, and perform our calculations in a general supersymmetry breaking scenario. We find that the prospects for dark matter are largely determined by the ratio of the universal gaugino mass $\left(m_{1 / 2}\right)$ to the the universal scalar mass $\left(m_{0}\right), \xi \equiv m_{1 / 2} / m_{0}$. For $\xi \ll 1$, both light $\left(<M_{W}\right)$ and heavy $\left(>M_{W}\right)$ dark matter candidates exist, while for $\xi \approx 1$ mainly the heavy candidates survive. For $\xi \gg 1$, consistency constraints of the model eliminate most dark matter candidates.
\end{abstract}

ACT-36

CERN-TH-6100/91

CTP-TAMU-31/91

May 1991

* Supported by an ICSC-World Laboratory Scholarship.

† Supported in part by DOE Grand DE-FG05-91-ER-40633. 


\section{Introduction}

Supersymmetric grand unification (SUSY GUTs) [1] has become fashionable again, since the accurate LEP measurements of $\sin ^{2} \theta_{w}$ and $\alpha_{s}$ show that ordinary GUTs are confirmed to be ruled out [2], while SUSY GUTs are quite viable. Of course, this latest piece of the puzzle should be taken together with the others that have turned up recently in connection with this general conceptual and calculational framework. Indeed, the LEP measurement of the number of light neutrino species and the (direct) lower and (indirect) upper [3] bounds on the top quark mass are well-known to be borne out very naturally in SUSY GUTs models with radiative electroweak symmetry breaking [4], correct prediction for the $m_{b} / m_{\tau}$ ratio [5], and perturbative unification [6].

Recently we have made a preliminary exploration of the prospects for dark matter [7] in the SUSY GUTs scenario ${ }^{*}$ [8]. Our previous paper concentrated more on the general methods to calculate accurately the cosmological relic abundance of the lightest neutralino $(\chi)$ (assumed to be the lightest supersymmetric particle (LSP)), while approximate expressions were used for the squark and slepton masses which influence the relic abundance results so dramatically. Also, only a specific supersymmetry breaking scenario was considered. In this paper we recalculate the relic abundance for the lightest neutralino using accurate relations for the sfermion masses which are valid in minimal SUSY GUTs models. We carry out this analysis in the most general supersymmetry breaking scenario. Our calculations involve a neutralino of generic composition and include all naturally allowed values of its mass. In addition, in computing the relic abundance [8], we consider all possible annihilation channels and do not use the approximation of effective lagrangians.

We first discuss the validity and accuracy of the usual supergravity mass formulae obtained by solving approximately the relevant renormalization group

* Prior analyses of dark matter candidates in supersymmetric models $[9,10,11,12]$ had incorporated only the relationship among the gaugino masses in SUSY GUTs models (except in $[11,12])$. 
equations (RGEs). Next we generalize the contribution to the relic abundance from the $\chi \chi \rightarrow t \bar{t}$ channel when non-negligible stop left-right mixing is present. Then we discuss the constraints on the parameter space of the model which follow from direct LEP searches for charginos, neutralinos, and sleptons, as well as the CDF search for squarks and gluinos. The mass formulae which we use imply certain consistency conditions which can be quite restrictive. Our results are rather remarkable in that the prospects for dark matter are determined to a large extent by the supersymmetry breaking scenario at high energies. We find that a scenario dominated by a universal scalar mass $\left(m_{0}\right)$ allows for quite light LSP dark matter candidates, while a scenario with comparable universal gaugino masses $\left(m_{1 / 2}\right)$ is only cosmologically interesting for heavier LSP masses. A gaugino mass dominated scenario appears to be disfavored.

\section{Supergravity mass relations}

For our present purposes, the supersymmetry breaking scenario can be given in terms of universal scalar $\left(m_{0}\right)$ and gaugino $\left(m_{1 / 2}\right)$ masses, and the hidden sector parameter $A$ [13]. For convenience we define the ratios $\xi \equiv m_{1 / 2} / m_{0}$ and $\xi_{A} \equiv A / m_{0}$. The gaugino masses $\left(M_{i}, i=1,2,3\right)$ at low energies are given by the solutions to their respective RGEs with the initial condition $M_{i}=m_{1 / 2}$ at $M_{X}$, and are given by $M_{i}=\left(\alpha_{i} / \alpha_{X}\right) m_{1 / 2}$, where $\alpha_{X}$ is the unified gauge coupling at $M_{X}$. In what follows we take $\alpha_{X}=0.0409$ and $M_{X} \approx 10^{16} \mathrm{GeV}$, which are derived from the following low-energy values [2]: $\alpha_{3}=0.111, \sin ^{2} \theta_{w}=0.233$, and $\alpha=1 / 127.9$; we also take $m_{t}=150 \mathrm{GeV}$. Thus $m_{1 / 2}=1.22 M_{2}=0.368 M_{3}$. The scalar masses are analogously obtained from the initial condition of a universal scalar mass $m_{0}$ at $M_{X}$. The squark and slepton mass RGEs can be solved exactly if the relevant Yukawa couplings can be neglected. For the first two generations the RGEs take the generic form (see e.g., $[4,11]$ )

$$
\frac{d m_{i}^{2}}{d t}=\frac{1}{8 \pi^{2}}\left(-\sum_{j=1}^{3} a_{i j} g_{j}^{2} M_{j}^{2}\right)
$$


where $t=\ln \left(\Lambda / M_{Z}\right)$. These can be solved exactly [14],

$$
\begin{aligned}
m_{i}^{2} & =m_{0}^{2}+m_{1 / 2}^{2} \sum_{j=1}^{3} \frac{a_{i j}}{2 b_{j}}\left(1-\frac{1}{F_{j}^{2}}\right)-M_{W}^{2} \frac{\tan ^{2} \beta-1}{\tan ^{2} \beta+1}\left[\left(T_{3 i}-Q\right) \tan ^{2} \theta_{w}+T_{3 i}\right] \\
& \equiv\left(1.22 M_{2}\right)^{2}\left(c_{i}+1 / \xi^{2}\right)+D_{i},
\end{aligned}
$$

where the $b_{i}$ are the one-loop beta function coefficients $\left(b_{3}=-3, b_{2}=1, b_{1}=\right.$ $33 / 5), F_{j} \equiv 1+\left(b_{j} \alpha_{X} / 2 \pi\right) \ln \left(M_{X} / M_{Z}\right), \tan \beta=v_{2} / v_{1}$ is defined as usual, $T_{3 i}$ and $Q$ are the isospin and electric charge of the sfermion, and the last piece in Eq. 2 comes from the D-terms in the potential. With the above numbers we get: $F_{1}=2.389, F_{2}=1.210, F_{3}=0.369$, and $c_{\tilde{e}_{L}, \tilde{\mu}_{L}, \tilde{\nu}}=0.514, c_{\tilde{e}_{R}, \tilde{\mu}_{R}}=0.150$, $c_{\tilde{u}_{L}, \tilde{c}_{L}}=c_{\tilde{d}_{L}, \tilde{s}_{L}}=6.134, c_{\tilde{u}_{R}, \tilde{c}_{R}}=5.720, c_{\tilde{d}_{R}, \tilde{s}_{R}}=5.670$.

For the third generation scalar masses, the RGEs receive additional contributions of the form $\alpha \lambda_{t}^{2} S_{t}+\beta \lambda_{b}^{2} S_{b}+\bar{\gamma} \lambda_{\tau}^{2} S_{\tau}(\alpha, \beta, \gamma \geq 0)$, where the $S_{t, b, \tau}$ are sums of squares of scalar masses and $A$ terms. For generic values of $\lambda_{t, b, \tau}$ these RGEs cannot be solved analytically (although a numerical solution is straightforward). Nevertheless, it is clear that the third generation low-energy sfermion masses will be smaller than their first and second generation counterparts due to the Yukawa coupling dependent terms added to the RGEs. In what follows we will basically neglect the effect of the Yukawa couplings, in which case the $c$ 's for the third generation sfermions are as given above. Note that for $\tilde{t}_{L, R}$, left-right mixing can be important, since the off-diagonal contribution to the stop squared mass matrix is given by

$$
m_{\tilde{t}_{L R}}^{2}=m_{t}\left(A_{t}-\mu / \tan \beta\right)
$$

where $\mu$ is the Higgs mixing parameter in the superpotential; the diagonal contributions (i.e., $m_{\tilde{t}_{L}}^{2}$ and $m_{\tilde{t}_{R}}^{2}$ ) are given in Eq. 2. The lighter mass eigenstate $\tilde{t}_{1}$ is

$\ddagger$ Rigorously speaking, the physical sfermion masses should be obtained by running the RGEs down to the sfermion mass $m_{i}$ and then solving the resulting implicit equation for $m_{i}$ [15].

* We do keep the top-quark mass in Eq. 2, i.e., $m_{\tilde{t}}^{2} \rightarrow m_{\tilde{t}}^{2}+m_{t}^{2}$. Also, the bottom and tau Yukawa couplings are not necessarily small in a model with $\tan \beta \gg 1$, and certainly not in superstring models [16]. 
lighter than all the other squarks and imposes nontrivial consistency constraints on the parameter space of the model, as discussed below.

\section{Effect of left-right mixing}

Since $\tilde{t}_{L, R}$ mixing can be sizeable, the computation of the contribution to the relic abundance of LSPs [8] from the $\chi \chi \rightarrow t \bar{t}$ annihilation channel must be upgraded to incorporate this effect. For brevity (details are given in [17]), here we just present an expression for $\tilde{a}_{t \bar{t}} \propto|\mathcal{M}(\chi \chi \rightarrow t \bar{t})|^{2}$ which arises in the computation of the relic abundance $\Omega_{\chi} h_{0}^{2}$, where $\Omega_{\chi} \equiv \rho_{\chi} / \rho_{\text {crit }}$, and $0.5 \lesssim h_{0} \lesssim$ 0.7 is a current estimate of the present value of the Hubble parameter in units of $100 \mathrm{~km} \mathrm{~s}^{-1} \mathrm{Mpc}^{-1}$ (see, e.g. the second Ref. of [7]); we thus favor the range $0.25 \lesssim \Omega_{\chi} h_{0}^{2} \lesssim 0.5$, while values of $\Omega_{\chi} h_{2}^{0}>1$ are cosmologically excluded. The result is

$$
\tilde{a}_{t \bar{t}}=\left[\sum_{i=1}^{2} \frac{1}{\Delta_{i}}\left(A_{i} m_{t}+\epsilon m_{\chi} B_{i}\right)\right]^{2},
$$

with $\Delta_{i} \equiv m_{\tilde{t}_{i}}^{2}+m_{\chi}^{2}-m_{t}^{2}$, and

$$
\begin{aligned}
& A_{i}=\sum_{\alpha=L, R}\left|G_{\alpha}^{\chi f \tilde{f}_{L}} U_{1 i}+G_{\alpha}^{\chi f \tilde{f}_{R}} U_{2 i}\right|^{2}, \\
& B_{i}=2 \operatorname{Re}\left(G_{L}^{\chi f \tilde{f}_{L}} U_{1 i}+G_{L}^{\chi f \tilde{f}_{R}} U_{2 i}\right)\left(G_{R}^{\chi f \tilde{f}_{L}} U_{1 i}+G_{R}^{\chi f \tilde{f}_{R}} U_{2 i}\right)^{*},
\end{aligned}
$$

where the $G_{L, R}^{\chi f \tilde{f}_{L, R}}$ couplings (and $\epsilon$ ) are defined in [8], and the orthogonal matrix $U$ rotates the $\tilde{t}_{L, R}$ states into the mass eigenstates $\tilde{t}_{1,2}$ through the angle $\theta$ defined by $\tan 2 \theta=2 m_{\tilde{t}_{L R}}^{2} /\left(m_{\tilde{t}_{L}}^{2}-m_{\tilde{t}_{R}}^{2}\right)$.

\section{Results and discussion}

We have computed the relic abundance of LSPs in the $\left(\mu, M_{2}\right)$ plane [8] for several choices of the parameters involved: $\xi, \xi_{A}, \tan \beta$, and the lightest Higgs boson mass $m_{h}$. We pick two representative values of $\tan \beta: 2$ and 8 . For these 
values we find that the $m_{h}$ dependence, which has-already been discussed in [8], does not affect the dark matter scenario significantly. Thus, we present our results for fixed $m_{h}=45 \mathrm{GeV}$. (This value of $m_{h}$ is allowed at tree-level for both values of $\tan \beta$.) As to the supergravity breaking parameters $\xi, \xi_{A}$, two choices have been used widely in the past: $m_{1 / 2} \gg m_{0}, A$, and $m_{1 / 2} \ll m_{0}, A$. We find that the value of $\xi_{A}$ does not affect the relic abundance results significantly, since in our approximation $A$ contributes only to $\tilde{t}_{L, R}$ mixing. (As discussed below, $A$ does restrict the parameter space due to its effect on the $\tilde{t}_{1}$ mass.) We then choose $\xi_{A}=1$ and $\xi=0.1,1.0,10.0$; our results are presented in Figs. 1-3.

\section{4a. Constraints on parameter space}

Direct experimental constraints: The LEP chargino mass lower bound $m_{\chi^{ \pm}}>$ $45 \mathrm{GeV}[18]$ and the ALEPH direct search for neutralinos [19] rule out substantial regions of parameter space (denoted by the 'LEP' lines and the 'ALEPH' regions in Figs. 1-3). The CDF lower bound on the gluino mass $m_{\tilde{g}}>150 \mathrm{GeV}$ [20] translates into $M_{2}>45 \mathrm{GeV}$ (denoted by the 'CDF' lines in Figs. 1-3).

Consistency constraints: The mass formulae given in Eq. 2 must always give $m_{i}^{2}>0$. Furthermore, the CDF lower bound on the squark mass $\left(m_{\tilde{q}}>100 \mathrm{GeV}\right.$ [20]) and that from LEP on the slepton mass $\left(m_{\tilde{l}}>43 \mathrm{GeV}\right.$ [21]) strengthen these consistency bounds further. In addition, by definition all squark and slepton masses must be greater than the LSP mass. These constraints give restrictions on the allowed $\left(\mu, M_{2}\right)$ parameter space that vary with the chosen values of $\xi$ and $\xi_{A}$. (Recall that $m_{\tilde{q}, \tilde{l}}$ vary throughout the $\left(\mu, M_{2}\right)$ plane due to their $M_{2}$ dependence, and in the case of $\tilde{t}_{1,2}$ its $\mu$ dependence as well.) However, generally one can observe that for small $\xi$ these bounds are automatically satisfied since $m_{i}^{2}=\left(1.22 M_{2}\right)\left(c_{i}+1 / \xi^{2}\right)+D_{i} \approx\left(1.22 M_{2} / \xi\right)^{2} \gg M_{2}^{2}$, while $m_{\chi} \lesssim \frac{1}{2} M_{2}$ in the region of interest (see e.g., Fig. 1 in [8]).

For larger values of $\xi$, what matters is the magnitude of $c_{i}$ and whether $D_{i}$ is negative or not. For the sneutrino, $c_{\tilde{\nu}}$ is small enough and $D_{\tilde{\nu}}<0$ so that there is a lower bound on $M_{2}$ to achieve $m_{\tilde{\nu}}>m_{\chi}$. This appears as a horizontal 
cutoff on the lower portion of the allowed region in Figs. 2b, 3a and 3b. Note that the effect is more important for larger $\tan \beta$, as expected from Eq. 2. For the $\tilde{e}_{R}, \tilde{\mu}_{R}, \tilde{\tau}_{R}$ masses, a similar phenomenon occurs since $c_{\tilde{e}_{R}, \tilde{\mu}_{R}, \tilde{\tau}_{R}}=0.150$ is so small, even though $D_{\tilde{e}_{R}, \tilde{\mu}_{R}, \tilde{\tau}_{R}}>0$. This effect appears as the voids in Fig. 3 at the upper left- and right-hand corners of the figures. One can easily understand the peculiar shape of these excluded regions by studying the LSP mass contours in those regions. Another important restriction comes from the $\tilde{t}_{1}$ mass, which must be larger than $\max \left\{100 \mathrm{GeV}, m_{\chi}\right\}$. Large values of $\mu$ drive the $\tilde{t}_{1}$ low (see Eq. 3) and are thus excluded (see voids in lower left- and right-hand corners of Figs. $2 \mathrm{a}$ and $3 \mathrm{a}$ ); this effect is weakened by large values of $\tan \beta$ and here is only visible for $\tan \beta=2$.

Other constraints: In a full dynamical calculation, the requirement of radiative electroweak symmetry breaking will correlate all the parameters of the model, essentially determining $\mu$ as a function of the supersymmetry breaking parameters, $\tan \beta$, and $m_{t}$. Also, in this scenario further experimental constraints (such as bounds on Higgs masses) could be imposed on the parameter space. Another set of constraints comes from direct and indirect dark matter searches. If the lightest neutralino populates the galactic halo, then neutralinos could be captured in the Earth and the Sun. In the latter case, neutralino annihilation would produce a flux of high energy neutrinos from the direction of the Sun, that could be observed in underground detectors. The non-observation of this flux puts bounds on the $\left(\mu, M_{2}\right)$ parameter space [22].

\section{4b. Relic density contours}

One can clearly see from Figs. 1-3 that the location of the cosmologically interesting regions, i.e., those which have $0.25 \lesssim \Omega_{\chi} h_{0}^{2} \lesssim 0.5$ and are represented by squares in Figs. 1-3, is weakly dependent on $\tan \beta$ and mostly determined by $\xi$. In the figures we have denoted by $\widetilde{B}, \widetilde{H}_{S(A)}$ regions of the $\left(\mu, M_{2}\right)$ plane where the LSP is mostly a pure bino or symmetric (antisymmetric) Higgsino [23,8]; any 
other regions are referred to as 'mixed'. In detail we find:"

(i) For $\xi=0.1$ (Figs. 1a,1b) these regions allow both light $\left(m_{\chi}<M_{W}\right)$ bino, Higgsino, and mixed dark matter candidates, as well as heavy $\left(m_{\chi}>M_{W}\right)$ bino candidates. Note that the light bino and mixed LSPs can be as light as the current experimental lower bound on the LSP mass $\left(m_{\chi} \gtrsim 20 \mathrm{GeV}\right)$ [24]. Going from $\tan \beta=2$ to $\tan \beta=8$ eliminates all light mixed dark matter candidates and reinforces the light bino ones. The small value of $\xi$ in this case gives large values for all sfermion masses $\left(m_{\tilde{q}, \tilde{l}} \gtrsim 500 \mathrm{GeV}\right)$ and this is why there is so much dark matter (stars in Fig. 1) for small $M_{2}$; these regions are excluded on cosmological grounds.

(ii) For $\xi=1.0$ (Figs. 2a,2b) the effects of the consistency constraints become visible. For $\tan \beta=2$ we can see the $\tilde{t}_{1}$ restriction, while for $\tan \beta=8$ we have mostly the $\tilde{\nu}$ restriction. The light mixed and very light bino allowed regions in Figs. 1a,1b disappear for both values of $\tan \beta$, but new light $\left(m_{\chi} \gtrless 60 \mathrm{GeV}\right)$ bino regions appear. The heavy bino regions remain, although shifted and enlarged a bit. The excluded regions for $\xi=0.1$ do not appear here since the sfermion masses are much lighter now.

(iii) For $\xi=10.0$ (Figs. 3a,3b) all bino dark matter disappears and we are left with only the ever-present small regions of light Higgsino dark matter. $\dagger \ddagger$ The $\tilde{\nu}^{\prime}$ and $\tilde{t}_{1}$ restrictions are more evident now, and the $\tilde{e}_{R}, \tilde{\mu}_{R}, \tilde{\tau}_{R}$ restrictions appear for heavy bino LSPs. This result agrees qualitatively with that in Ref. [11], where $m_{1 / 2} \gg m_{0}, A$ was assumed.

* There are small patches of missing data points in Figs. 1-3 (not to be confused with the voids arising from the consistency restrictions on parameter space discussed above). These occur for $m_{\chi} \approx \frac{1}{2} m_{A}\left(m_{A}=115(47) \mathrm{GeV}\right.$ for $\left.\tan \beta=2(8)\right)$ and correspond to a pole in the annihilation channel $\chi \chi \rightarrow A \rightarrow b \vec{b}[8]$; for these points our calculational approach fails [8].

$\dagger$ The results in our previous paper [8] were derived for $\xi \gg 1$, but in actuality correspond here to a case between $\xi=1.0$ and $\xi=10.0$, due to the approximate relations used for the sfermion masses in [8]. Also, no consistency constraints were imposed in that paper.

$¥$ The light Higgsino regions remain mostly unchanged for fixed $\tan \beta$ and varying $\xi$ since the LSP couples to $f-\tilde{f}$ very weakly $\left(\propto m_{f}\right)$ in these regions. 


\section{Conclusions}

We have presented an appraisal of the prospects for neutralino dark matter in minimal SUSY GUTs models with generic supersymmetry breaking. On cosmological grounds, our results favor models with equal or larger values of the universal scalar mass $m_{0}$ relative to the universal gaugino mass $m_{1 / 2}$. Quite light and experimentally accesible dark matter candidates are obtained in this class of models. Our computations are as accurate as possible short of a full dynamical evolution of the model parameters from high to low energies.

Calculations relevant for direct detection of dark matter particles have already been attempted in the SUSY GUTs scenario [25], while those relevant for indirect detection through neutralino annihilation in the Sun have not. In this latter context, it is interesting to note that the experimental bounds on the ensuing high-energy neutrino flux from the Sun constrain only rather mildly LSP particles which are mostly pure bino or Higgsino admixtures (favored by our analysis); this is not true for more generic (i.e., mixed) LSP compositions [22]. 


\section{REFERENCES}

1. See e.g., G. G. Ross, Grand Unified Theories, (Benjamin/Cummings, MA, 1983); C. Kounnas, A. Masiero, D. V. Nanopoulos and K. A. Olive, Grand Unification With and Without Supersymmetry and Cosmological Implications, (World Scient. Publ. Comp., Singapore, 1984).

2. J. Ellis, S. Kelley and D. V. Nanopoulos, Phys. Lett. B 249 (1990) 441 and Phys. Lett. B 260 (1991) 131; U. Amaldi, W. de Boer, and H. Fürstenau, CERN preprint CERN-PPE/91-44 (1991).

3. J. Ellis and G. Fogli, Phys. Lett. B 249 (1990) 543; V. Barger, J. Hewett, and T. Rizzo, Phys. Rev. Lett. 65 (1990) 1313; P. Langacker and M.-X. Luo, University of Pennsylvania preprint UPR-0466T (1991).

4. A. B. Lahanas and D. V. Nanopoulos, Phys. Rep. 145 C (1987) 1.

5. A. J. Buras, J. Ellis, M. K. Gaillard, D. V. Nanopoulos, Nucl. Phys. B 135 (1978) 66; D. V. Nanopoulos and D. A. Ross, Nucl. Phys. B 157 (1979) 273, Phys. Lett. B 108 (1982) 351, and Phys. Lett. B 118 (1982) 99.

6. L. Durand and J. L. Lopez, Phys. Lett. B 217 (1989) 463 and Phys. Rev. D 40 (1989) 207.

7. For reviews on dark matter and its detection, see e.g., V. Trimble, Ann. Rev. Astron. Astrophys. 25 (1987) 425; J. R. Primack, B. Sadoulet, and D. Seckel, Ann. Rev. Nucl. Part. Sci. 38 (1988) 751; D. O. Caldwell, Mod. Phys. Lett. A 5 (1990) 1543; P. F. Smith and J. D. Lewin, Phys. Rep. 187 C (1990) 203.

8. J. L. Lopez, D. V. Nanopoulos, and K. Yuan, Texas A \& M University preprint CTP-TAMU-13/91.

9. J. Ellis, J. S. Hagelin, D. V. Nanopoulos, K. A. Olive and M. Srednicki, Nucl. Phys. B 238 (1984) 453; J. Ellis, J. Hagelin, and D. V. Nanopoulos, Phys. Lett. B 159 (1985) 26; K. Griest, Phys. Rev. D 38 (1988) 2357; 
Phys. Rev. D 39 (1989) 3802(E); K. A. Olive and M. Srednicki, Phys. Lett. B 230 (1989) 78 and Nucl. Phys. B 355 (1991) 208; K. Griest, M. Kamionkowski, and M. S. Turner, Phys. Rev. D 41 (1990) 3565; J. Ellis, L. Roszkowski, and Z. Lalak, Phys. Lett. B 245 (1990) 545; L. Roszkowski, Talk given at the UCLA International Conference on Trends in Astroparticle Physics, November 1990; K. Yuan, PhD Thesis Texas A\&M University (1991).

10. R. Flores, K. A. Olive and D. Thomas, Phys. Lett. B 245 (1990) 509; K. Olive and D. Thomas, Nucl. Phys. B 355 (1991) 192.

11. J. Ellis and F. Zwirner, Nucl. Phys. B 338 (1990) 317.

12. M.M. Nojiri, KEK preprint KEK-TH-263 (September 1990).

13. For reviews see: R. Arnowitt and P. Nath, Applied $N=1$ Supergravity (World Scientific, Singapore 1983); H. P. Nilles, Phys. Rep. 110 C (1984) 1; and Ref. [4]

14. C. Kounnas, A. Lahanas, D. V. Nanopoulos, and M. Quirós, Nucl. Phys. B 236 (1984) 438.

15. See e.g., G. Gamberini, G. Ridolf, and F. Zwirner, Nucl. Phys. B 331 (1990) 331; A. Faraggi, J. Hagelin, S. Kelley, and D. V. Nanopoulos, Texas A \& M University preprint CTP-TAMU-22/91.

16. See e.g., J. L. Lopez and D. V. Nanopoulos, Texas A \& M University preprint CTP-TAMU-27/91, and references therein.

17. K. Yuan, in preparation.

18. L3 Collaboration, B. Adeva et. al., Phys. Lett. B 233 (1989) 530; ALEPH Collaboration, D. Decamp et. al., Phys. Lett. B 236 (1990) 86; OPAL Collaboration, M. Z. Akrawy et. al., Phys. Lett. B 240 (1990) 261.

19. ALEPH Collaboration, D. Decamp et. al., Phys. Lett. B 244 (1990) 541. 
20. CDF Collaboration, F. Abe et. al., Phys. Rev. Lett. 62 (1989) 1825; S. Geer, talk presented at the First Int. Symposium on Particles, Strings, and Cosmology (PASCOS-90), Boston, March 27-31, 1990.

21. Particle Data Group, Phys. Lett. B 239 (1990) 1.

22. See e.g., G. Gelmini, P. Gondolo, and E. Roulet, Nucl. Phys. B 351 (1991) 623 and SISSA preprint No. SISSA-88/89-EP; M. Kamionkowski, Fermilab preprint FERMILAB-Pub-90/181-A.

23. K. Olive and M. Srednicki in Ref. [9].

24. L. Roszkowski, Phys. Lett. B 252 (1990) 471.

25. J. Ellis and R. Flores, CERN preprint CERN-TH.6063/91.

\section{FIGURE CAPTIONS}

1) The relic abundance contours for $m_{h}=45 \mathrm{GeV}, \xi_{A}=A / m_{0}=1, \xi=$ $m_{1 / 2} / m_{0}=0.1$, and (a) $\tan \beta=2$ and (b) $\tan \beta=8$. The meaning of the six different symbols is: (1) dots $\left(\Omega_{\chi} h_{0}^{2}<0.025\right)$; (2) crosses $\left(0.025<\Omega_{\chi} h_{0}^{2}<\right.$ $0.1)$; (3) diamonds $\left(0.1<\Omega_{\chi} h_{0}^{2}<0.25\right)$; (4) squares $\left(0.25<\Omega_{\chi} h_{0}^{2}<0.5\right)$; (5) vertical crosses $\left(0.5<\Omega_{\chi} h_{0}^{2}<1\right)$; (6) stars $\left(\Omega_{\chi} h_{0}^{2}>1\right)$. Regions marked by stars are cosmologically excluded, while those marked by squares correspond to theoretically preferred values of $\Omega_{\chi} h_{0}^{2}$. In the dotted areas the relic abundance is too small to account even for dark matter in galactic halos. The voids correpond to violations of consistency constraints of the model. We apply the following constraints: (i) $m_{\chi_{1}^{ \pm}}>45 \mathrm{GeV}$ (marked as 'LEP'), (ii) $m_{\tilde{g}}>150 \mathrm{GeV}$ (marked as 'CDF'), $m_{\tilde{q}}>100 \mathrm{GeV}, m_{\tilde{l}}>$ $43 \mathrm{GeV}$, and (iii) unsuccessful neutralino searches (marked by 'ALEPH'), as described in the text.

2) Same as Fig. 1, but with $\xi=1.0$.

3) Same as Fig. 1, but with $\xi=10.0$. 


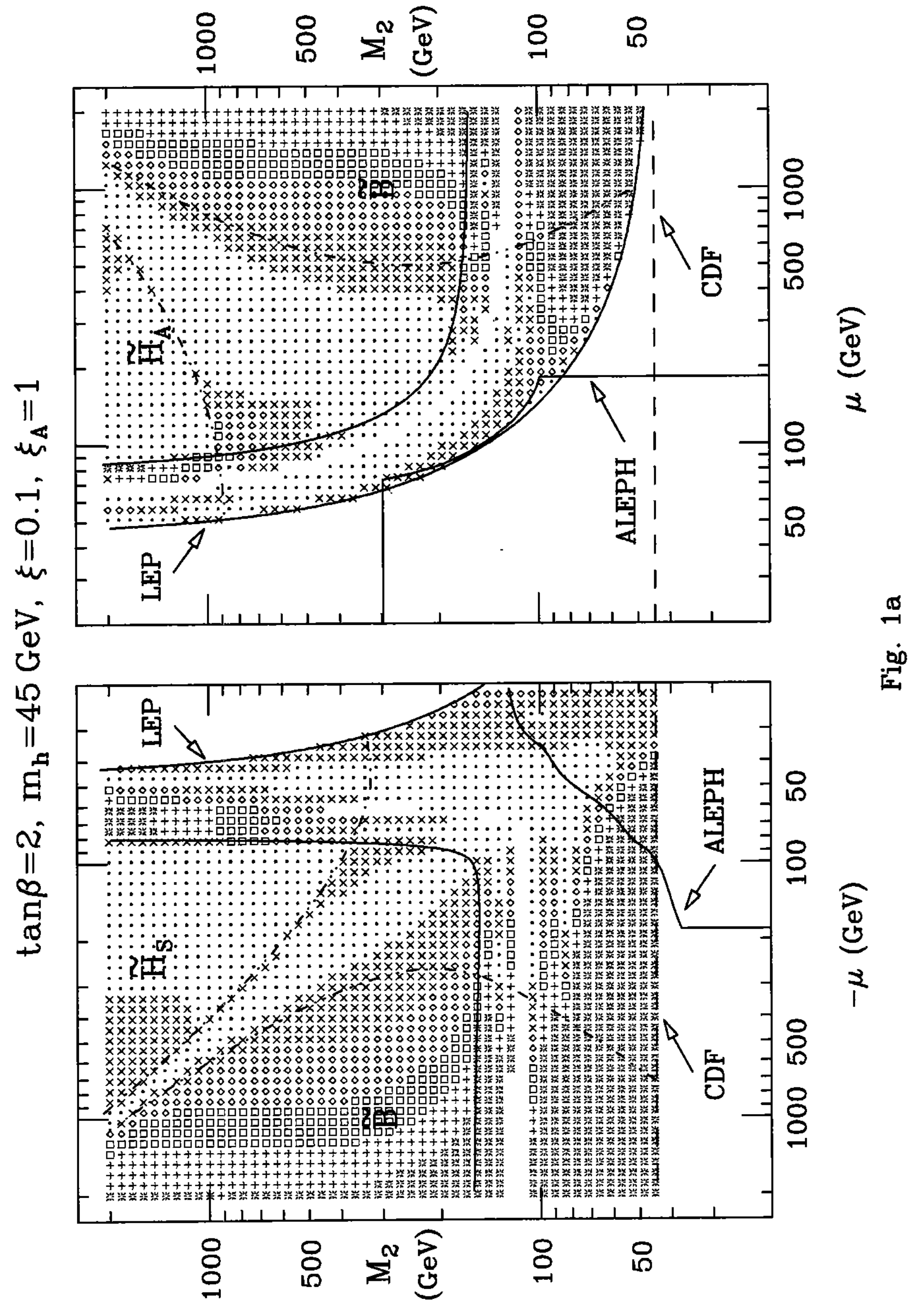




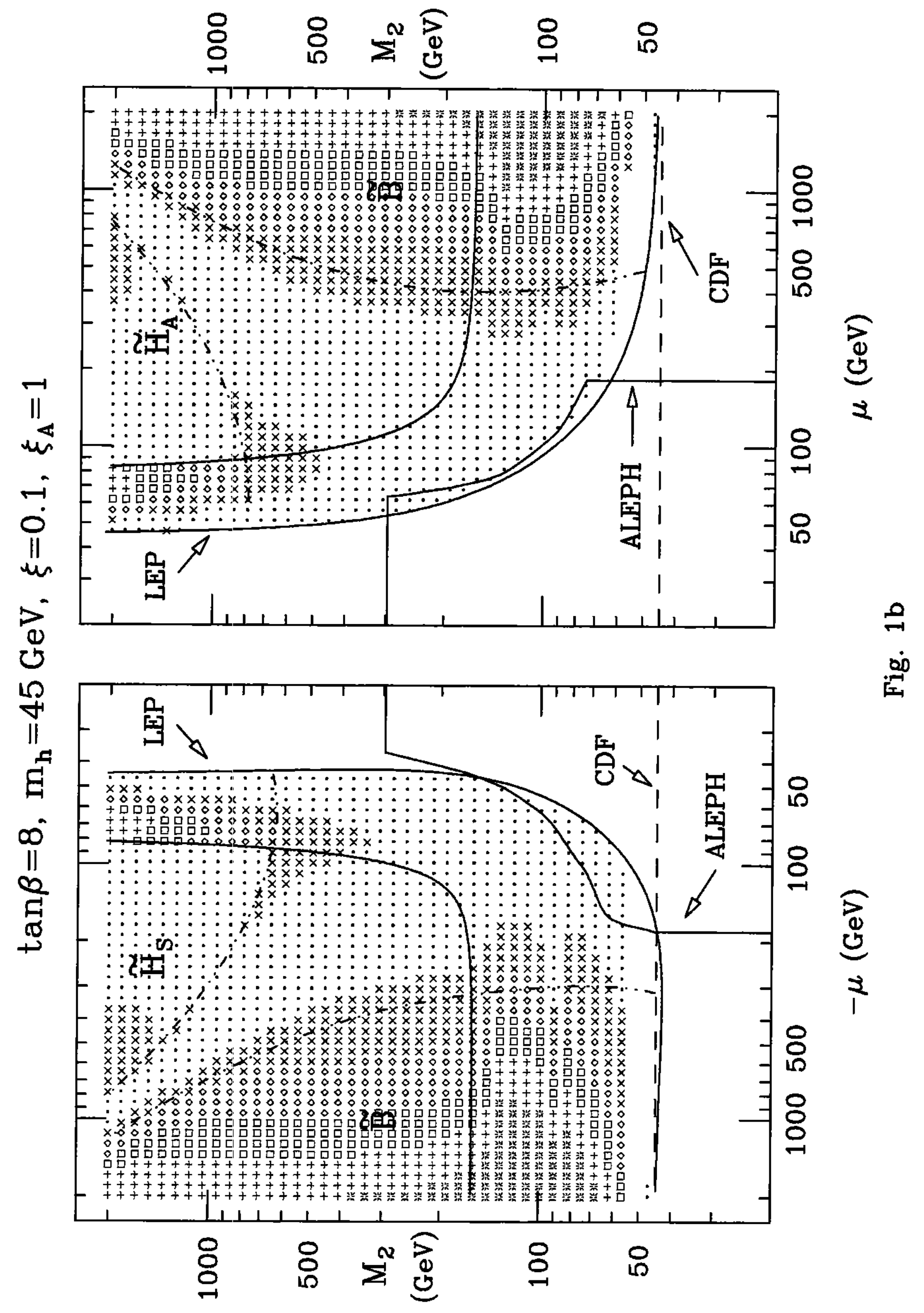




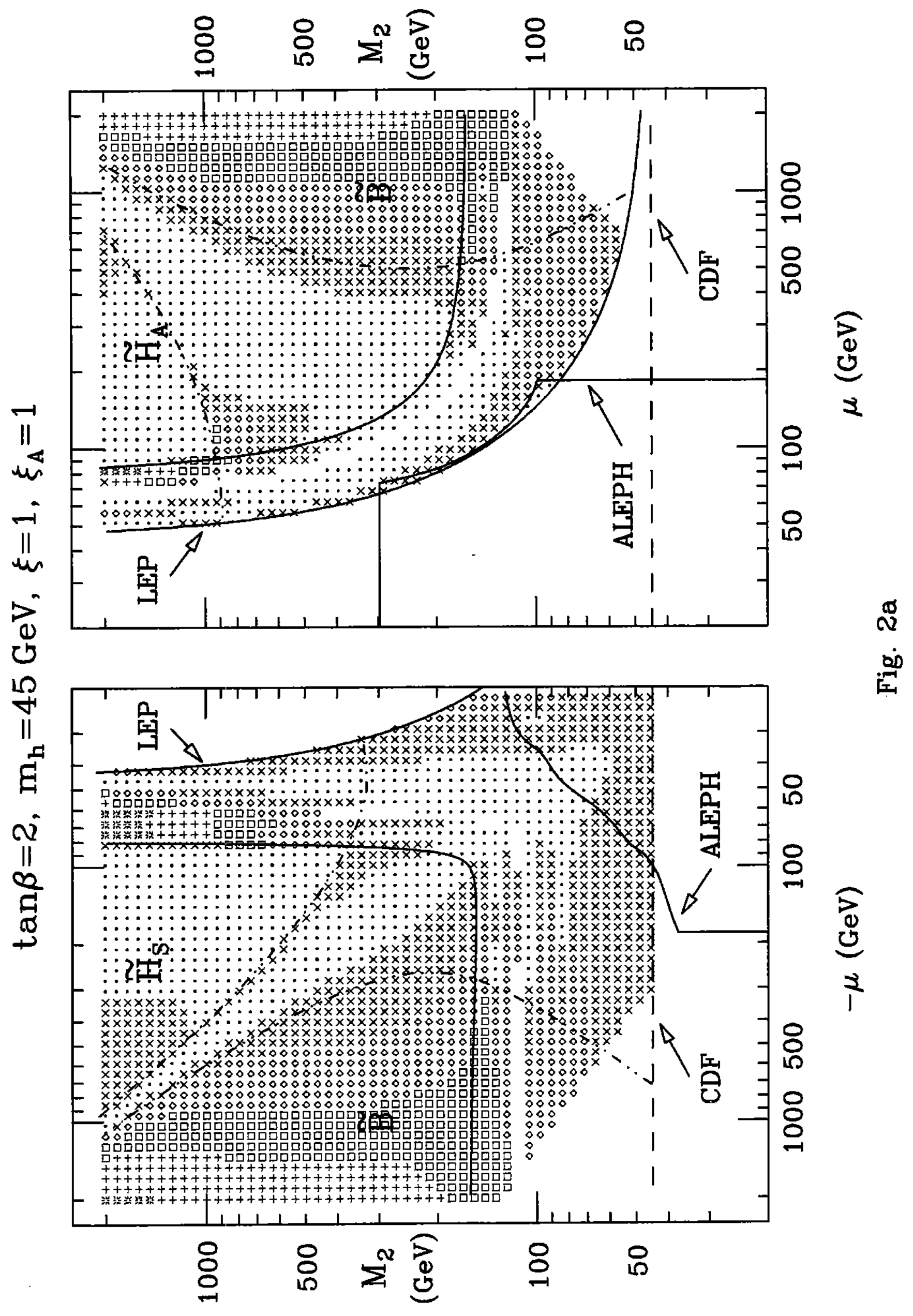




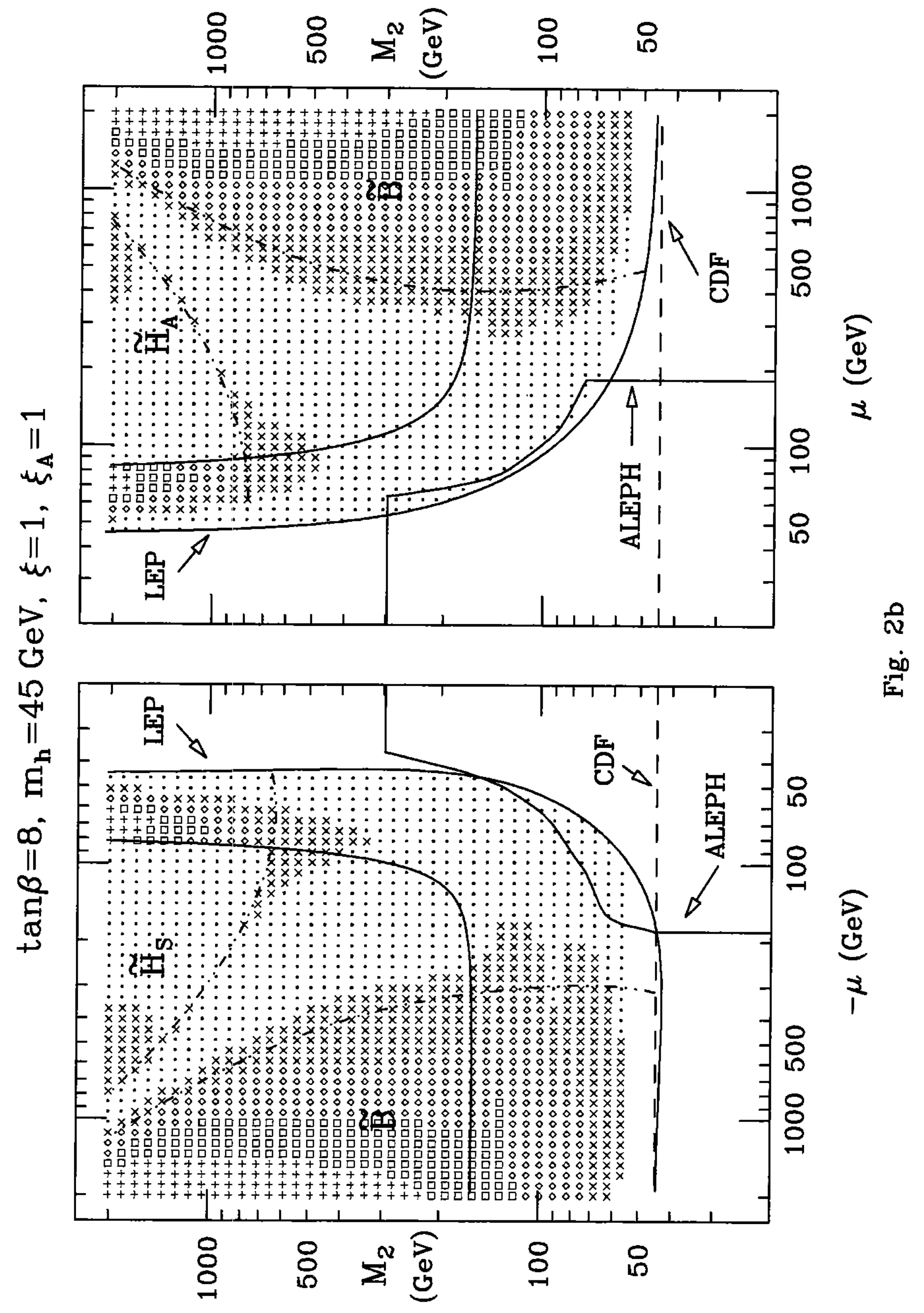









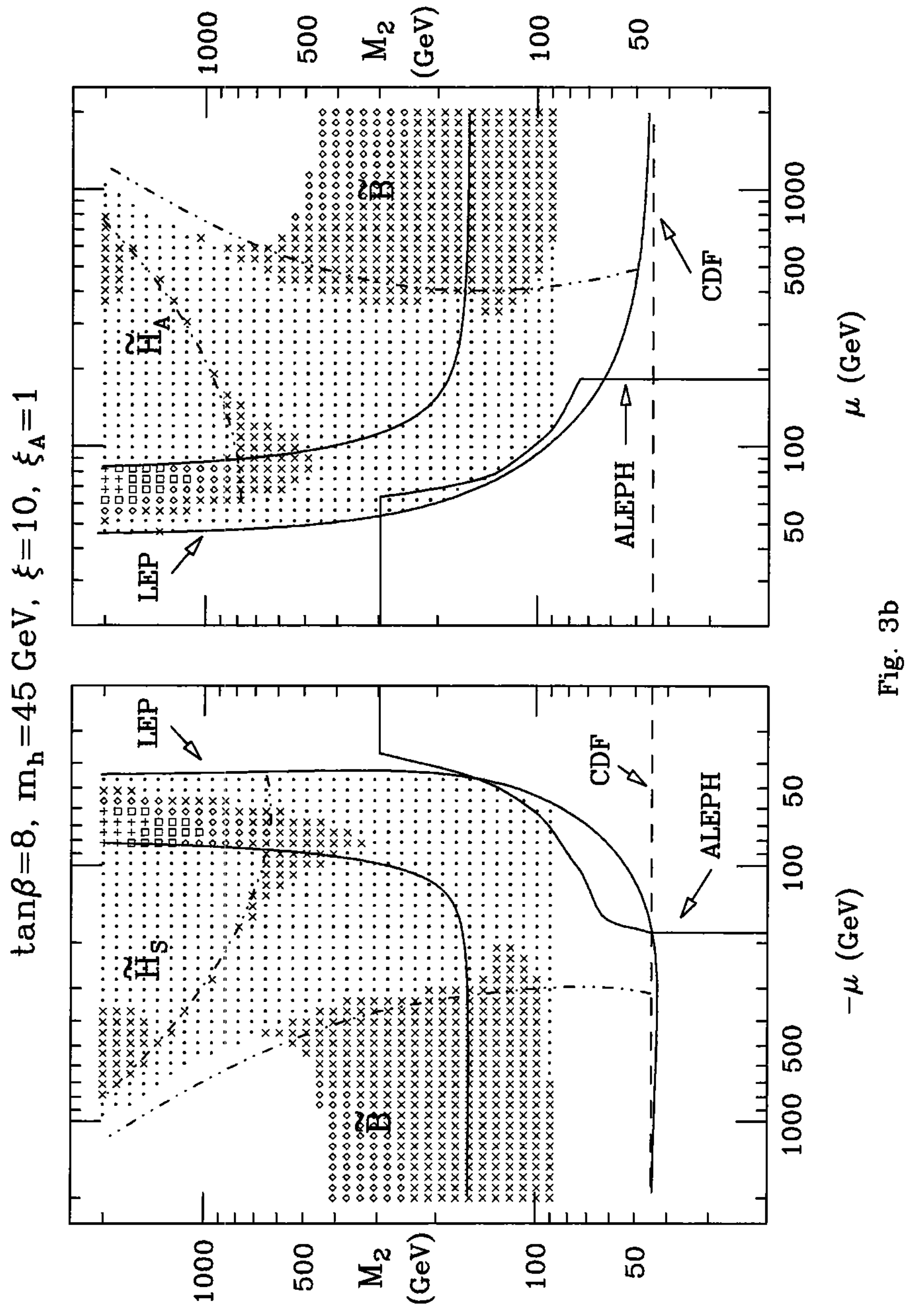

